\title{
A Framework for Self-Enforced Optimal Interaction Between Connected Vehicles
}

\author{
Marcin Stryszowski ${ }^{\circledR}$, Stefano Longo ${ }^{\circledR}$, Senior Member, IEEE, Dario D’Alessandro, \\ Efstathios Velenis, Senior Member, IEEE, Gregory Forostovsky, and Sabato Manfredi ${ }^{\circledR}$, Member, IEEE
}

\begin{abstract}
This paper proposes a decision-making framework for Connected Autonomous Vehicle interactions. It provides and justifies algorithms for strategic selection of control references for cruising, platooning and overtaking. The algorithm is based on the trade-off between energy consumption and time. The consequent cooperation opportunities originating from agent heterogeneity are captured by a game-theoretic cooperativecompetitive solution concept to provide a computationally feasible, self-enforced, cooperative traffic management framework.
\end{abstract}

Index Terms-Connected cars, game theory platooning, negotiation, overtake, V2V.

\section{INTRODUCTION}

$\mathbf{T}$ ODAY'S paradigm of road vehicle operation is far from optimal: individual human drivers compete for space without means to understand one another's intentions. They lack understanding of fuel-efficient vehicle operation and are vulnerable to fatigue. As a result, the velocity profiles are very variable, saturated with many deceleration and acceleration events and high peak velocities. All of these maneuvers cause demand for oversized powertrains, waste energy and increase air pollution.

The largest contributors to the energy inefficiency are breaking and aerodynamic drag. The former can be minimized by the elimination of traffic uncertainty. The latter, since drag is roughly proportional to the square of velocity, can be addressed by careful optimal selection of the cruising velocity. The proposed framework provides an algorithm for the selection of optimal maneuvers in a cooperative manner. Neglecting tire slip losses, which occur at the limit of handling, any maneuver can be viewed as a velocity transient. Velocity, in turn is a trade-off between time elapsed and energy efficiency. An optimization algorithm defines a cost function, with a minimizer as optimal cruising velocity. It is followed

Manuscript received October 25, 2018; revised February 15, 2019, April 30, 2019, and July 24, 2019; accepted April 13, 2020. This work was supported in part by the Engineering and Physical Sciences Research Council (EPSRC) and in part by Arrival Ltd. The Associate Editor for this article was H. A. Rakha. (Corresponding author: Marcin Stryszowski.)

Marcin Stryszowski, Stefano Longo, and Efstathios Velenis are with the Advanced Vehicle Engineering Centre, Cranfield University, Cranfield MK43 0AP, U.K. (e-mail: m.stryszowski@ cranfield.ac.uk; s.longo@ cranfield.ac.uk; e.velenis@cranfield.ac.uk).

Dario D'Alessandro and Sabato Manfredi are with the Department of Electrical Engineering and Information Technology (DIETI), University of Naples Federico II, 80138 Naples, Italy (e-mail: dario.dalessandro@studenti.unina.it; smanfred@unina.it).

Gregory Forostovsky is with Arrival Ltd., London W1G 0EG, U.K. (e-mail: gregory@arrival.com).

Digital Object Identifier 10.1109/TITS.2020.2988150 by a scenario where the Ego Vehicle (EV), while cruising at its optimal velocity, encounters an Obstacle Vehicle (OV) ahead. Today, the solution is to either merely platoon behind, losing time, or waiting for an overtake window of unknown size, wasting energy and compromising safety. Information exchange, however, enables understanding of the other agent's objectives and provides means to find a mutually optimal strategy. The condition of self-enforcement removes the need for legislative effort.

The literature indicates clarity in the direction road transport is heading: the emergence of driverless and automated traffic [1] is a matter of time. Such vehicles will be equipped with high computational power and machine-based situational awareness, which shall be supplied not only by the onboard sensors, but also by means of cooperative perception and negotiation [2] mediated by vehicle-to-vehicle (V2V) and vehicleto-infrastructure (V2I) wireless communication systems [3]. Given that these technologies are rapidly developing [4], information exchange is assumed to be complete and perfect, meaning no information is lost.

Very narrow scope of research addresses the problem of optimal cruise velocity selection [5], [6]. Both view velocity as a trade-off between energy and time, but introduce a subjective, abstract parameter to control the bias.

Platooning, where vehicles follow one another, has been considered from traffic stability [7] and cooperative sensing [2] perspective. The decrease of aerodynamic drag has also received considerable attention [8]. However, there is no research addressing the multi-agent dynamics of platoon formation.

Autonomous overtaking has received a lot of attention already. Research focuses on the theoretical background to guide further development and harmonization of the lateral and longitudinal controls or the technical requirements to handle it [9], [10]. More recent studies propose a division of the maneuver into three phases to apply adaptive control algorithm [11], or application of spacecraft rendezvous algorithms to approach the problem [12]. Most importantly, [13] studies the feasibility of autonomous overtaking performed by Model Predictive Control, taking the safety and comfort as objectives. The cost function is defined to penalize deviation from the reference velocity and trajectory, taking into account the distance to the oncoming vehicle. However, the formulated method assumes no cooperativity and does not track energy consumption or time. The application of automated driving technology by a number of companies [14], [15] indicates 
a necessity for studies on the energy efficiency of scenarios which such vehicles may encounter.

There are a number of approaches to cooperation on intersections, either centralized [16], [17], or decentralized [18]; with hardware demonstration ready [5]. Cooperation is facilitated by numerous intersection management schemes [19]-[21]. All of these publications consider the throughput of intersections, while neglecting agents' individual preferences and self-enforcement, so are dedicated for high-density traffic. Additionally, a vehicle may change lanes, or merge into an intersections - conflict problems which fit into a game theoretic formulation with promising results [22]-[24].

Game Theory has has also found application in hybrid powertrain control systems to predict user behaviour [25] or distributed control for unmanned aerial vehicle formation [26].

All state-of-the-art traffic optimization frameworks assume agents to be homogeneous, neglecting the differences between them and thus the negotiation aspect. While the threat of congestion makes such arrangement self-enforceable, it is not optimal. The authors consider the heterogeneity and emergent capability for negotiation derived from the properties of nonzero sum games. This approach captures also the human, individualistic aspect of the problem [27]-[30] to offer a novel, user-centric framework for negotiation of traffic scenarios between connected traffic agents in low and medium intensity traffic. The negotiation in a distributed topology requires means of payment to enforce it, what has been proposed in a form of a traffic intersection algorithm [21] and a block-chain based mechanism is being implemented [31].

The problem is formulated to find optimal references, considering the resources managed: energy consumed by vehicles and value of time of users.

Research on Connected Autonomous Vehicles (CAV) lacks studies on the selection of optimal velocity. When the decision is only constrained by safety speed limits, the velocities might be much higher than necessary from the energy perspective. We propose that cruising velocity selected is a trade-off between the cost of vehicle operation, and the time understood as the user's value of time as an opportunity cost [32]. This notion is analogous to the Airline Cost Index used as decision-making factor in aircraft operation, which, due to its confidential nature cannot be used to automated Air Traffic Control [33].

While optimisation algorithms are employed for single decision agents, as two self-optimizing agents meet, the conflict between agents' objectives is modelled by a game theory. An explicit, self-enforced solution concept is employed to formulate the agent intentions into a single optimisation problem. The agents are thus assumed to be honest, which eliminates the safety-critical risk of adversary's defect and allows for fine refinement of the solution, by means of cooperativecompetitive (co-co) solution concept [34]. The co-co solution eliminates also the need for finding Nash equilibrium, which belongs to NP-hard complexity class [35].

This paper consists of five Sections. Section II presents an outline of the framework developed. Section III proposes the mathematical formulation and assumptions on which it is developed. Section IV provides simulation results and

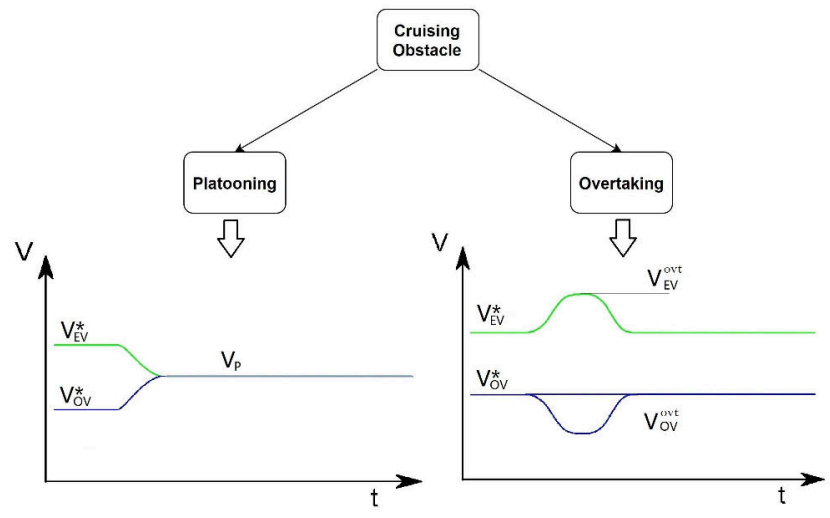

Fig. 1. Decision tree for a scenario where a slower vehicle ahead is encountered. A decision whether to overtake or platoon is negotiated and optimal velocity profiles are selected from energy optimality point of view.

Section $\mathrm{V}$ concludes the paper discussing the results and further work.

\section{The Automated Negotiation Framework}

The conceptualized framework is geared to balance vehicle's resource utilization, that is both energy and user's time, in accordance with the user-specified objective. With cost function defined, the decision objectives is to minimize it. The simplest scenario is a free cruise, where vehicle maintains an optimal cruising velocity $V^{*}$. Then the cruising interruption scenario: a slower vehicle ahead can be resolved by either platooning or an overtake, according to the decision tree presented in Fig. 1. Given that platooning causes a delay and excessive speed and overtaking costs energy, but mitigates the delay, the best agreeable maneuver strategies are found: platooning velocity $V_{P}$, or overtake velocity profiles $V_{n}^{O V T}$ and the cheapest variant is selected. The payment $p$ is exchanged to facilitate cooperation and share the benefit.

This framework also carries a capability to empower future users of CAV to adjust their operating strategy based on journey's objective, providing an added value not only of refined energy optimization, but also a sense of system's agility, beyond that of a public transport system [36]. The users would be able to define their cost of time delay, thereby influencing the cost function's profile. It may provide a sense of control and ownership over the vehicle, possibly mitigating an important factor deterring people from CAV ownership [37].

\section{Problem Formulation}

The proposed framework assumes that all vehicle operation consists of cruising or handling cruising obstacle, either joining a platoon or performing an overtake. With these assumptions in mind, we can identify the input parameters required, listed in Tab. I, and the decision variables for each event, which are listed in Tab. II.

While refined vehicle models improve result precision, the main objective of the paper is to propose and study dynamics of applied negotiation algorithm. Simplistic vehicle models are thus applied for clarity, as there is little novelty 
TABLE I

INPUT PARAMETERS

\begin{tabular}{ccc} 
Scenario & Input & Symbol \\
\hline \hline Cruise & User's value of time & $C_{T}$ \\
\hline Platooning & expected platooning distance & $S_{S}[\mathrm{~m}]$ \\
\hline Overtake & dynamic overtake gap (see Appendix A) & $S_{\max }[\mathrm{m}]$ \\
\multicolumn{3}{c}{ TABLE II } \\
ConTROL VARIABLES \\
Scenario & Decision variable & Symbol \\
\hline \hline Cruise & Equilibrium velocity & $V^{*}$ \\
\hline Platooning & Consensus vel., payment & $V_{P}^{\#}[\mathrm{~m} / \mathrm{s}], p[\$]$ \\
\hline Overtake & Cons. vel. profiles, payment & $V_{E V}^{o v t}, V_{O V}^{o v t}, p$
\end{tabular}

in already researched powertrain models [38]. The powertrain model is defined in Appendix A

Each vehicle has a set of constants defining it: mass $m_{n}$, rolling resistance $\mu_{\text {roll }, n}$, aerodynamic area $A$, drag coefficient $C_{d}$, powertrain efficiency $\eta_{P}$, unit cost of energy source $C_{E, n}$ and longitudinal distance $X_{S}$, from which the energy consumption is found. At the beginning of every journey, the user selects his personal cost of time delay $C_{T, n}$.

\section{A. Game-Theoretic Solution Concept}

In the proposed approach, two or more self-optimizing agents meet with different, but not mutually exclusive objectives. Thanks to properties described below, agents may then agree on a globally optimal solution and share the benefit, according to their negotiative power. Equilibrium seeking approaches to GT do not require enforcement, but are computationally expensive and cannot guarantee the payoff-dominant solution [35]. In this approach, a GT is used to break the multiagent problem into an optimisation problem. It is incentivised by a promise of optimal solution, a self-enforced cooperation can be achieved by a cooperative-competitive (co-co) solution concept [34], which guarantees the Pareto-optimal equilibrium at low computational cost. The threat of cost wasted by a maneuver interrupted by the adversary's agreeability constraint and promise of efficiency justify the choice of the solution concept. The problem of the system's sensitivity to agent honesty is not straightforward and is discussed in Appendix C.

If agents are heterogeneous, their interaction is a non-zero sum game. If they are willing to communicate, they can adopt a pair of strategies $\left(u_{1}^{\#}, u_{2}^{\#}\right)$ which minimize the combined, cooperative cost $J_{\text {sum }}^{\#}$ as

$$
J_{\text {sum }}^{\#}=\min _{u_{1}, u_{2}}\left(J_{1}\left(u_{1}, u_{2}\right)+J_{2}\left(u_{1}, u_{2}\right)\right)
$$

where $J_{\text {sum }}^{\#}$ is the total, combined cost of cooperative strategies.

In the pair of strategies yielding $J_{\text {sum }}^{\#}$, one of the agent's best strategy $J_{n}^{*}$ may be better, however, than $J_{n}^{\#}$, which is not agreeable unless an incentive is provided. The benefiting agent needs to provide a side payment $p=J^{b}$ to compensate, guaranteeing under threat of rejection, that

$$
J_{n}^{\#}+J_{n}^{b}>J_{n}^{*} .
$$

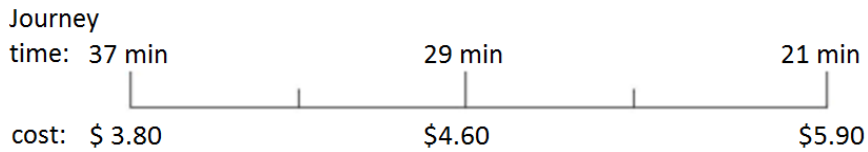

Fig. 2. Proposed slider with example values of the cruise time and cost, to interface user's intention with the system.

As proposed in [39], the payment should be proportional to the players' power (relative values of $J_{n}^{*}$ ), and contribution to the common achievement. The payoffs are divided as follows:

$$
\begin{aligned}
& J_{\text {split }}^{\#}=\frac{J_{1}\left(u_{1}, u_{2}\right)+J_{2}\left(u_{1}, u_{2}\right)}{2}, \\
& J_{\text {split }}^{\text {b }}=\frac{J_{1}\left(u_{1}, u_{2}\right)-J_{2}\left(u_{1}, u_{2}\right)}{2} .
\end{aligned}
$$

With such division, we can split the game as a sum of a purely cooperative game. Where players have payoffs $J_{n}^{\#}$, and a purely competitive, zero-sum game, where players have opposite payoffs $J_{n}^{b}=-J_{-n}^{b}$. Then, the result of a co-co game is defined as

$$
J_{1}=\frac{J^{\#}}{2}+J^{b}, \quad J_{2}=\frac{J^{\#}}{2}-J^{b} .
$$

\section{B. Cruising}

The cost of opportunity is intended to be selected by the user of the vehicle, as a mean of expressing their intention as to how much they value their time and can be viewed as value of time $C_{T}$. It is defined by the user together with the destination of the journey and can be interfaced by a trade-off slider as in Fig. 2.

If we then consider that the intention is to move a distance $\Delta S$, in some steps $\delta S$, and $V$ is the rate of movement, then, since velocity cannot be infinite, there is always a time elapsed $\Delta T$ defined as

$$
\Delta T=\frac{\Delta S}{V} .
$$

Since the loss of time occurs over the distance of the journey

$$
\Delta T=\frac{1}{V}:=\int_{0}^{S} \frac{\delta S}{V} d S .
$$

The energy is found in a similar manner from the propulsive force necessary to maintain a steady state velocity $v$, considering the powertrain efficiency $\eta_{P}$, aerodynamic characteristics $C_{d} A$ and rolling resistance coefficient $\mu_{\text {roll }}$

$$
E(v)=\frac{1}{\eta_{P}} \int_{0}^{S} \frac{1}{2} \rho_{\text {air }} C_{d} A v^{2}+g \mu_{\text {roll }} v d S .
$$

Then the value of the unit of energy consumed by the powertrain is defined as $C_{E}$, and the overall cost function of each agent $n$ is then a balance between time and energy

$$
\begin{aligned}
c J_{n}(v) & =C_{E} E(v)+C_{T} \Delta T \\
& =A v^{2}+B v+C+\frac{D}{v},
\end{aligned}
$$

where:

$$
\begin{aligned}
A & =\frac{C_{E}}{\eta_{P}} \frac{1}{2} \rho_{a i r} C_{d} A \\
B & =\frac{C_{E}}{\eta_{P}} g \mu_{\text {roll }} \\
C & =0 \quad D=C_{T} .
\end{aligned}
$$




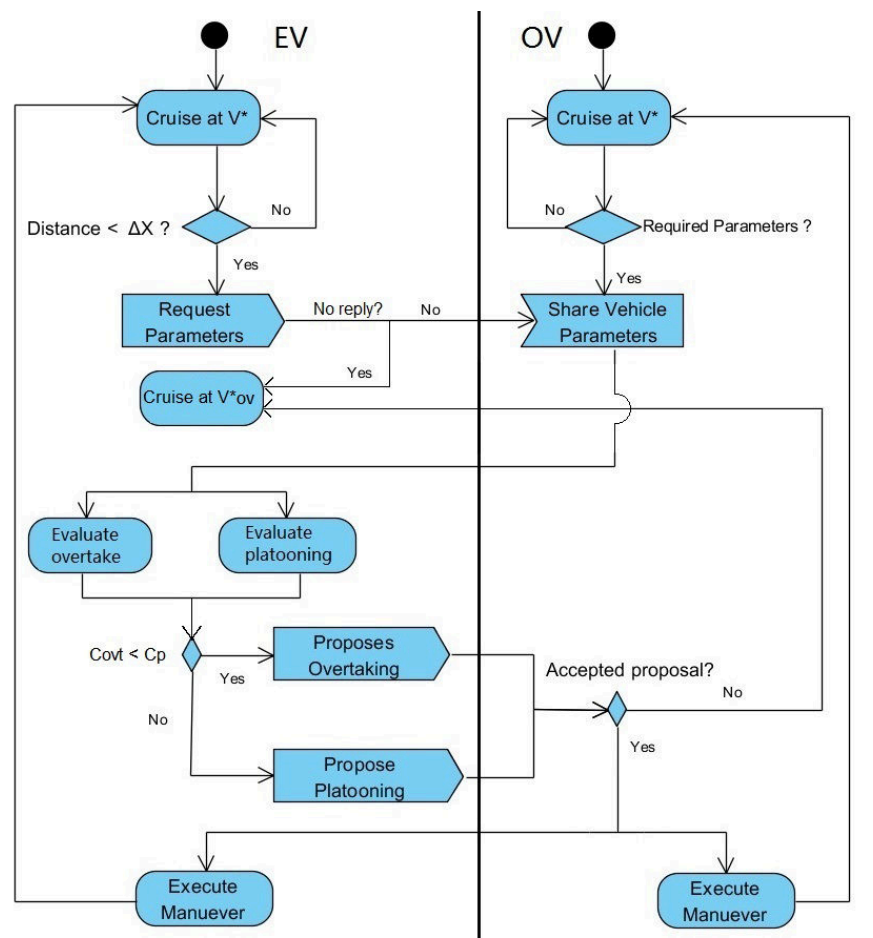

Fig. 3. Activity diagram of the communication needed to find agreeable strategy.

The optimal cruise velocity $V^{*}$ which minimizes the cost function is then found as

$$
V^{*}=\underset{v}{\operatorname{argmin}}\left(J_{n}(v)\right)
$$

The minimal cost per unit distance, i.e. the cruising cost $C_{C}$, corresponding to $V^{*}$, is defined as

$$
C_{C, n}=J_{n}\left(V_{n}^{*}\right)=\min _{v} J_{n}(v) .
$$

The cost function is convex given that the derivative of the cost function (10) with respect to velocity assumes the form

$$
\frac{d J_{n}}{d v}=2 A v+B-\frac{D}{v^{2}}
$$

which, when solved for $V \in \mathbb{R}^{+}$has only one root, guaranteeing a unique minimum.

\section{Cooperative Interaction Handling}

With the cost function for cruising velocity proposed above, the heterogeneity of agents makes the co-co solution capable to enable cooperation without enforcement. Fig. 3 presents the activity diagram of the algorithm.

As an agent meets an obstacle, it requests adversary's preference parameters and finds the best maneuver, which is proposed and agreed upon or rejected, limiting the negotiation communication to four messages.

\section{Platooning}

If a cruising vehicle encounters a slower vehicle or a platoon ahead, one of the solutions is to request an increase of the velocity, incentivizing it with payment. The proposed approach, assumes that vehicles share their local cost function
TABLE III

Platooning Payoff Matrix $M_{P, n}$

\begin{tabular}{c|cccc}
$V_{P}$ & $V_{O V}^{*}$ & $V_{O V}^{*}+\Delta V$ & $\cdots$ & $V_{E V}^{*}$ \\
\hline$V_{O V}^{*}$ & $j_{n}\left(V_{O V}^{*}\right)$ & $N a N$ & $\cdot$ & $\cdot$ \\
$V_{O V}^{*}+\Delta V$ & $N a N$ & $j_{n}\left(V_{O V}^{*}+\Delta V\right)$ & $N a N$ & $\cdot$ \\
$\vdots$ & $\cdot$ & $N a N$ & $\ddots$ & $N a N$ \\
$V_{E V}^{*}$ & $\cdot$ & $\cdot$ & $N a N$ & $j_{n}\left(V_{E V}^{*}\right)$
\end{tabular}

$j_{n}(v)$, which defines the cost of departure from $V^{*}$ and is defined as

$$
\left.j_{n}(v)\right|_{V_{O V}} ^{V_{E V}}=J_{n}(v)-C_{C, n},
$$

or, as the minus cruise $\operatorname{cost} C_{C, n}$ value defining constant $C$ (10c). The local cost functions $j_{E V}(v)$ and $j_{O V}(v)$, for platooning velocities between $V_{E V}^{*}$ and $V_{O V}^{*}$, when discretized with a step $\Delta V$, form a payoff matrix $M_{P, n}$, as in Tab. III. It defines the influence of agents strategies on each other. Since any set of strategies outside of the diagonal of the matrix would be infeasible from a safety perspective, information loss is neglected and the solution concept enables precise, cooperative strategy selection, values not on the diagonal are rejected.

The payoffs from diagonals of $M_{P, E V}$ and $M_{P, O V}$ are then summed as

$$
j_{P}(v)=j_{E V}(v)+j_{O V}(v)
$$

and the optimal platooning velocity $V_{P}$ is found as

$$
C_{P}=\min _{V_{P}} j_{P}(v),
$$

subject to agreeability constraint

$$
0 \leq j_{E V}\left(V_{P}\right)-j_{O V}\left(V_{P}\right) .
$$

Then, the initiating agent proposes a platooning velocity $V_{P}$. The cost of the OV's departure from $V_{O V}^{*}$ is covered by the EV's payment $p_{P}=j_{O V}\left(V_{P}\right)$.

To evaluate the cost of agreeability, the unconstrained minimum is defined as

$$
C_{P}=\min _{V_{P}^{N C}} j_{P}\left(V_{P}^{N C}\right)
$$

$V_{P}^{N C}$ is the unconstrained minimizer. The Price of Anarchy $(P o A)$, defining the loss of optimality of the GT solution in relation to the globally optimal, is then

$$
\text { PoA }=\frac{j_{P}\left(V_{P}\right)}{j_{P}\left(V_{P}^{N C}\right)} .
$$

The root of the gradient of $j_{P}(v)$ provides the unconstrained minimizer

$$
V_{P} \ni \nabla j_{P}\left(V_{P}\right)=0 .
$$

The agreeability constraint is a line $V_{a g r}$ found on the intersection of local cost functions

$$
V_{a g r} \ni j_{E V}\left(V_{a g r .}\right)=j_{O V}\left(V_{a g r}\right) .
$$




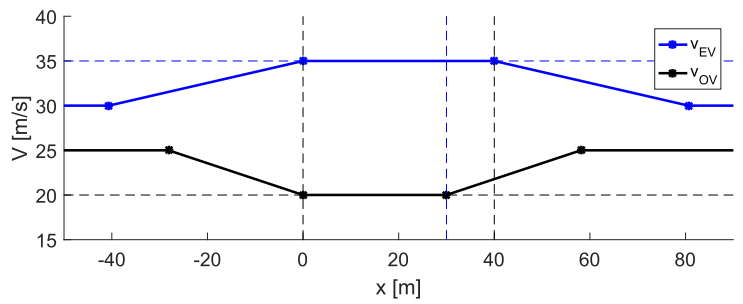

Fig. 4. A simplified overtake model in space domain.

Then, if $V_{a g r}<V_{P}$

$$
C_{P}=\min _{V_{P}} j_{P}\left(V_{a g r}\right)
$$

\section{E. Overtaking Manoeuvre}

An overtake is an alternative to the platooning strategy. The economy behind it is to spend energy in order to mitigate the continuous loss of time when platooning. An energy-focused overtake model is proposed.

1) Overtake Model: The overtake manoeuvre occurs when the distance between vehicles $\Delta X$ is within the absolute value of the sum of their safety distances

$$
X_{s}=X_{S, E V}+X_{S, O V}<|\Delta X|
$$

from $-X_{s}$ with the $\mathrm{OV}$ in the lead, to $+X_{s}$ with the $\mathrm{EV}$ in the lead. The total relative distance change during maneuver is $2 X_{s}$.

The longitudinal distance of manoeuvre is constrained by the maximal distance available, $S_{\max }$, when alongside. Then, the distance of the $\mathrm{OV}$ is constrained to

$$
S_{\max , O V}=S_{\max }-\left(2 X_{S}\right) .
$$

It is assumed that the manoeuvre is performed either at a constant velocity $v_{\text {ovt }, n}$ or constant acceleration $a_{n}$. The selection of maneuver accelerations is dependent on numerous control variables of the vehicle, including battery wear, subjective user comfort, and safety. In order to focus on the negotiation algorithm, we have decided to keep $a_{n}$ fixed. The factors in the cost function is the energy and is balanced with time. So each agent's decision variable is the the overtake velocity. Example velocity profiles are presented in Fig. 4.The dynamics control is neglected, as it has already been addressed [13].

The overtake then consists of three phases: acceleration at $a_{n}$ from $V_{n}^{*}$ to the preselected overtake velocity $V_{\text {ovt }, n}$, cruising at constant $V_{\text {ovt }, n}$ and deceleration back to $V_{n}^{*}$ at $-a_{n}$. Acceleration rates are assumed constant, leaving $V_{\text {ovt }}$ the only decision variable.

Then, the powertrain model returns energy consumption to evaluate the energy expense $C_{E, n}^{o v t}$. The overtake cost is found by adding the time cost as

$$
J_{o v t}\left(V_{E V}^{o v t}, V_{O V}^{o v t}\right)=\sum_{N} C_{E, n}^{o v t}+C_{\Delta T, n}^{o v t} .
$$

The manoeuvre is constrained by the oncoming traffic

$$
V_{E V}^{o v t}-V_{O V}^{o v t}>2 X_{s} \frac{V_{E V}^{o v t}}{S_{\max }} .
$$

TABLE IV

Payoff Matrix $M_{V_{\text {ov }}}$ For an Overtake. The Position $M_{1, N}$ Is PReSENTED AS InFEASIBLE. THE COLUMN AND Row With $V_{n}^{*}+\Delta V$ IS NEGLECTED FOR CLARITY

\begin{tabular}{c|ccc}
$V_{E V}^{\text {ovt }} \backslash V_{O V}^{\text {ovt }}$ & $V_{O V}^{\max }$ & $\cdots$ & $V_{O V}^{*}$ \\
\hline$V_{E V}^{*}$ & $J_{o v t}\left(V_{E V}^{*}, V_{O V}^{\text {ovt }}\right)$ & $J_{o v t}\left(V_{E V}^{\text {ovt }}, V_{O V}^{\text {ovt }}\right)$ & $N a N$ \\
$\cdot$ & $\cdot$ & $J_{\text {ovt }}\left(V_{E V}^{\text {ovt }}, V_{O V}^{\text {ovt }}\right)$ & $J_{\text {ovt }}\left(V_{E V}^{\text {ovt }}, V_{O V}^{\text {ovt }}\right)$ \\
$V_{E V}^{\max }$ & $J_{\text {ovt }}\left(V_{E V}^{\max }, V_{O V}^{\max }\right)$ & $\cdots$ & $J_{\text {ovt }}\left(V_{E V}^{\max }, V_{O V}^{*}\right)$
\end{tabular}

Since the overtake gap is never stationary, a maximal distance to perform an overtake is derived in Appendix B. Given a gap of $S_{\text {gap }}$ moving from the opposite direction at $V_{\text {gap }}$

$$
S_{\text {max }}=S_{\text {gap }}\left(1-\frac{V_{\text {gap }}}{V_{E V}^{\text {ovt }}}\right) \text {. }
$$

This can be viewed as relative velocity being sufficient to pass before the EV's distance exceeds $S_{\max }$. It is assumed that vehicles coming from the opposite direction have a known, constant velocity, so the $S_{\text {gap }}$ is constant.

2) Optimal Overtake: With the overtake model as described above a matrix of possible solutions $M_{V_{\text {oot }}}$ is created. Given a velocity set from $V_{n}^{*}$ to maximum overtake velocity $V_{n}^{\max }$ discretized with a step $\Delta V$

$$
V_{n}^{\text {ovt }}=\left\{V_{n}^{*}, V_{n}^{*}+\Delta V, \ldots, V_{n}^{\max }\right\},
$$

the $M_{V_{\text {oot }}}$ assumes the form as in Tab. IV.

Then the optimal overtaking manoeuvre is a vector of $V_{o v t, n}$ which minimizes $M_{V_{\text {ovt }}}$

$$
C_{\text {ovt }}\left(V_{E V}, V_{O V}\right)=\min _{V_{n}^{\text {oot }}}\left(M_{V_{\text {oot }}}\left(V_{E V}, V_{O V}\right)\right) .
$$

$V_{\text {ovt }, n}$ is found by finding a root of cost function's gradient

$$
\left(V_{E V}^{o v t}, V_{O V}^{o v t}\right) \ni \nabla J_{o v t}\left(V_{E V}^{o v t}, V_{O V}^{o v t}\right)=0,
$$

subject to feasibiliity constraint

$$
V_{O V}^{o v t}<-\frac{2 X_{s}}{S_{\text {max }}\left(1-\frac{V_{g a p}}{V_{E V}^{o o t}}\right)} V_{E V}^{o v t}+V_{E V}^{o v t}
$$

and powertrain force constraint

$$
F_{n e t, n}<\hat{F}_{n},
$$

where $\hat{F}_{n}$ is the maximal propulsive force.

Finally, as agreeability condition, payment $p_{o v t}=C_{O V}^{o v t}$, covering all costs of the manoeuvre on OV's side is issued, as the EV would be free to cruise unimpeded.

\section{F. Decision Rule}

Having evaluated the cost of platooning $C_{P}$ and the cost of overtake $C_{\text {ovt }}$, the decision to overtake is selected if

$$
C_{P} S_{S} \geq C_{\text {ovt }}
$$

and platooning otherwise. The EV proposes to OV a feasible strategy and a payment, which, to ensure enforcement, must satisfy (2), that is for a maneuver $x$ the strategy is accepted if

$$
C_{x}-p \geq C_{C}
$$


TABLE V

Vehicle Types and Their Differentiating Parameters

\begin{tabular}{c|ccccc}
$\begin{array}{c}\text { Veh. type } \\
\text { Symbol }\end{array}$ & $\begin{array}{c}\text { mass } \\
m[\mathrm{~kg}]\end{array}$ & $\begin{array}{c}\text { aerodyn. area } \\
A\left[\mathrm{~m}^{2}\right]\end{array}$ & $\begin{array}{c}\text { drag coef. } \\
C_{d}[-]\end{array}$ & $\begin{array}{c}\text { length } \\
\Delta X[\mathrm{~m}]\end{array}$ & $\begin{array}{c}\max \text { force } \\
\hat{F}_{n}[\mathrm{kN}]\end{array}$ \\
\hline Car & 1400 & 2 & 0.3 & 10 & 4 \\
Truck & $10^{4}$ & 4 & 0.5 & 25 & 10
\end{tabular}

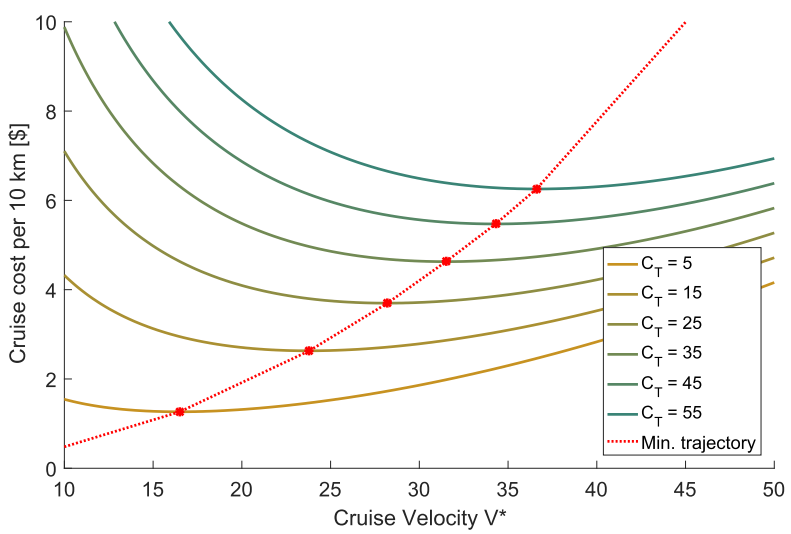

Fig. 5. Preference functions for various costs of time. Red curve indicates the trajectory of the functions' minima, increasing steeply with value of time.

\section{Simulation Examples}

In order to verify the performance of the framework, some typical scenarios are analysed. Two vehicle types are considered: a car and a truck. Their individual parameters are listed in Tab. V. Common parameters are: powertrain efficiency, assumed $\eta_{P}=0.82$, rolling resistance $\mu_{\text {roll }}=0.005$, and energy weight, corresponding to its market value $C_{E}=0.12$ $[\$ / \mathrm{kWh}][40]$. In the overtake problem the acceleration is $a_{n}=2[\mathrm{~m} / \mathrm{s}]$ and the model dynamics is discretized with a timestep $\Delta t=0.1[\mathrm{~s}]$. To model the cost of an overtake, the integration of the velocity profile is required at every iteration. This slows down the numerical optimization algorithm. Together with the intended embedded implementation using Objective-C language, it has lead us led to select an exhaustive search for optimization. With the precision of $0.01[\mathrm{~m} / \mathrm{s}]$ the solution takes on average 5.2 [s], however, assuming that the solution is on the feasibility constraint, what is justified by the convexity of the cost function, it takes 0.57 [s].

\section{A. Example 1 - Cruising Velocity Selection}

The sensitivity of optimal cruising velocity $V^{*}$ to user's objective $C_{T}$ is examined. Fig. 5 presents several preference functions for Car's $V P$ and the trajectory of the minimizer, for $C_{T}$ varying between $\$ 5$ and $\$ 55$.

\section{B. Example 2 - Negotiation of Platooning for Varying Vehicle Parameters and User Objectives}

The evaluation of departure costs from optimal velocity and possible solution points are visualized in Fig. 6. Point 1 marks the cost of a baseline, non-cooperative maneuver, point 2 the minimal agreeable cost and point 3 the Pareto optimal cost, which is not feasible under agreeability constraint, as the payment required to enforce it outweighs the benefit.

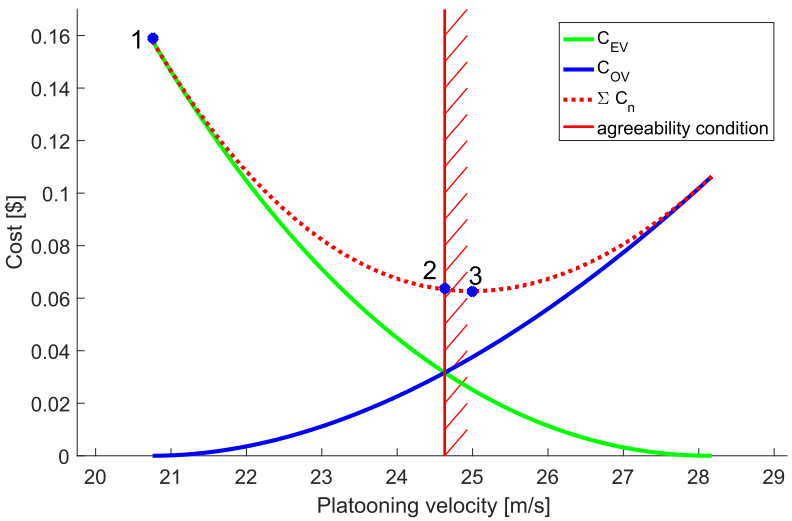

Fig. 6. Matching agents' preferences, to negotiate the optimal strategy for a $5 \mathrm{~km}$ platooing. Point 1 is the noncooperative cost, point 2 the agreeable, and point 3 is the optimal.

TABLE VI

Vehicle Type And Preferences for Each CASE

\begin{tabular}{c|cc|cc|cc} 
Feature $\backslash$ Case & \multicolumn{2}{|c}{$\mathrm{C} 1$} & \multicolumn{2}{c}{$\mathrm{C} 2$} & \multicolumn{2}{c}{$\mathrm{C} 3$} \\
& EV & OV & EV & OV & EV & OV \\
\hline Veh. type & Car & Truck & Car & Car & Truck & Car \\
$C_{T}[\$ / \mathrm{h}]$ & 25 & 67 & 25 & 10 & 167 & 10
\end{tabular}

TABLE VII

Platooning Solution Costs AND Prices of ANARChy (18). In CASE 1 Optimum Is Within CONSTRAint

\begin{tabular}{c|ccc|cc} 
& \multicolumn{3}{|c}{ Cost $\left[\$ 10^{-2}\right]$} & \multicolumn{2}{c}{ Price of Anarchy } \\
Case & NonC. & Agreeable & Pareto & NonC. & Agreeable \\
\hline 1 & 15.7 & - & 12.7 & 1.23 & 1 \\
2 & 15.7 & 6.31 & 6.26 & 2.5 & 1.008 \\
3 & 105 & 12 & 9.6 & 10.9 & 1.247
\end{tabular}

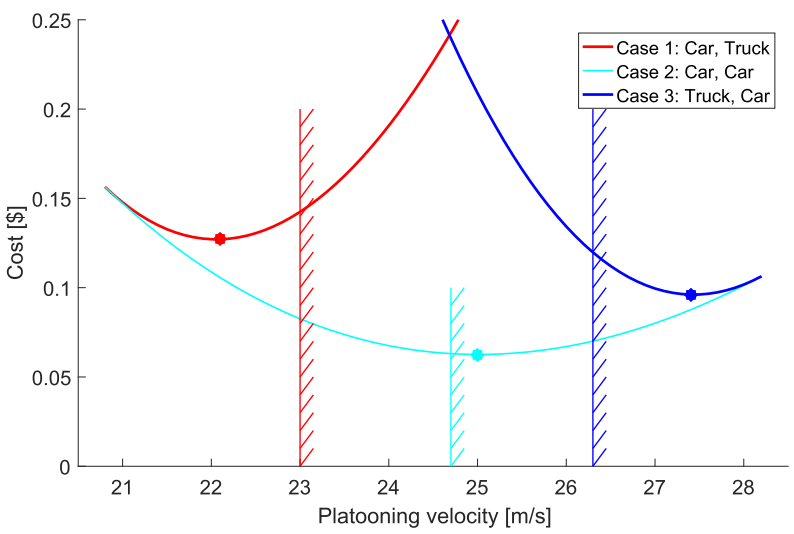

Fig. 7. Sum of platooning cost for $5 \mathrm{~km}$ for each case. Vertical line marks the agreeability constraint. Observe the negotiation power adjusting to the agent type. The case 1 is the only case where the optimum is within constraint limit. Points mark the functions' minima.

The same calculation has been performed for various vehicle types and $C_{T}$ selected to match $V^{*}$ for different vehicle types, as in Tab. VI. The results are presented in Fig. 7 and Tab. VII.

Case 1 considers a scenario where a fast car encounters a slower truck. Case 2 analyses two cars, and Case 3 a hurrying truck encountering a slower car. Cooperation yields considerable savings, while agreeability constraint causes minimal PoA. 


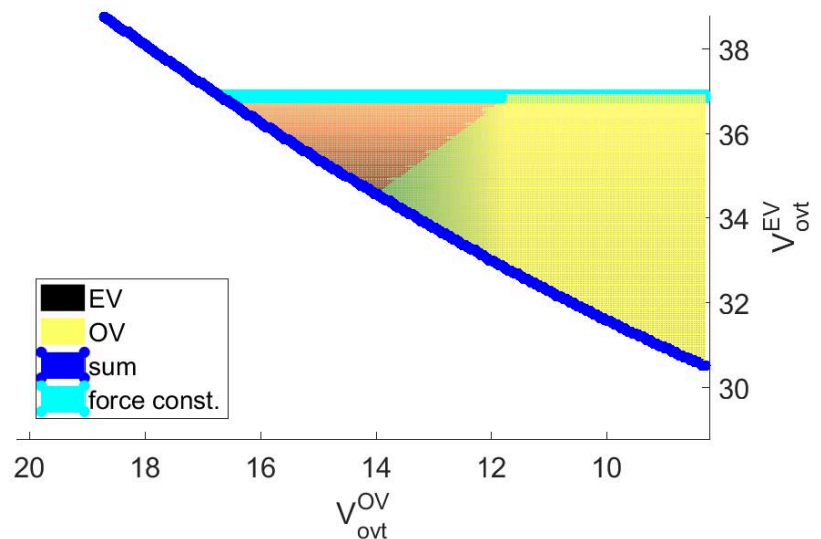

Fig. 8. A projection of the above figure from above, showcasing the constrained solution space.

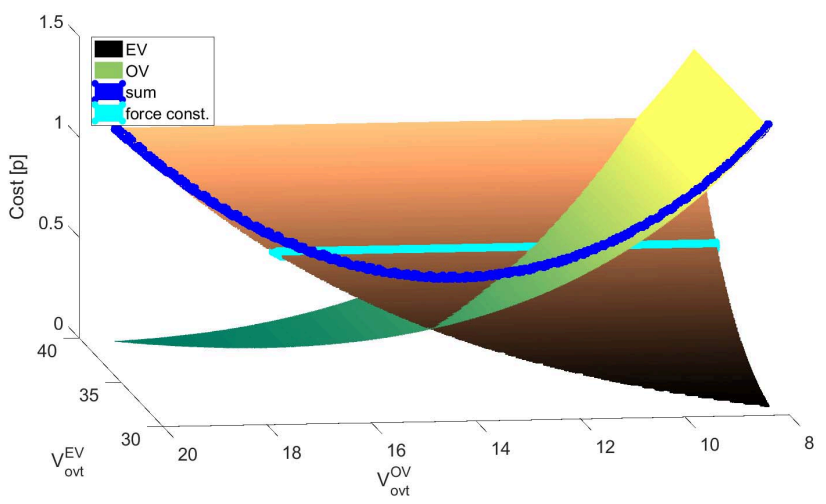

Fig. 9. An example of overtake costs surfaces and the feasibility front of their sum, on which optimal maneuver parameters are found.

\section{Example 3 - Overtaking Maneuver}

For the same vehicles as in Example 1, the values of $M_{V_{n}^{\text {ovt }}}$ assume the distribution presented in Fig. 9. For small overtake velocity differences $\left(V_{E V}^{o v t}-V_{O V}^{o v t}\right)$, the maneuvers are rejected as infeasible. The cost increases quickly, leaving the feasibility boundary as location of the optimal solution. The constraint on propulsive force rules out solutions for which required acceleration is not achievable at given $V^{\text {ovt }}$. In the example presented in Fig. 8 outlines the projection of the surface of feasible solutions. The constraint is at $V_{E V}^{o v t}=36.9[\mathrm{~m} / \mathrm{s}]$. An isometric view of the result is presented in Fig. 9. The optimal solution for an overtake gap of $80[\mathrm{~m}]$ is found to be, in this example, $V_{E V}^{o v t}=34.7[\mathrm{~m} / \mathrm{s}]$ and $V_{O V}^{o v t}=14.3[\mathrm{~m} / \mathrm{s}]$.

\section{Example 3 - Sensitivity of Overtake to Distance Available}

Evaluation of an optimal overtake cost as a function of distance available has been performed. Three cases from Tab. VI are being considered and results presented in Fig. 10. The overtake velocities required and total cost of maneuver are plotted. The cost of maneuver decreases hyperbolically with overtake gap available.

\section{E. Raspberry Pi Algorithm Implementation}

In order to demonstrate the practical applicability of the proposed framework the algorithm has been implemented in a

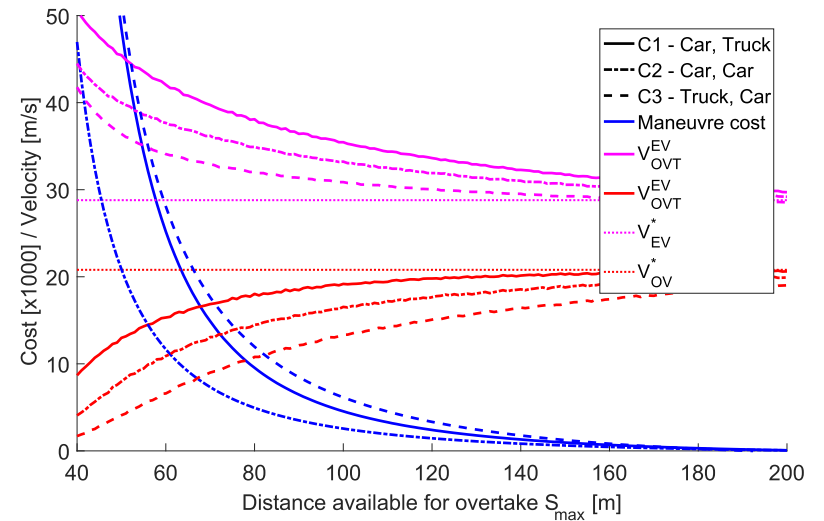

Fig. 10. Overtake cost, and payment required for performing an overtake for changing space available for the maneuver. In range from 40 to $200 \mathrm{~m}$. Oncoming velocity is $20 \mathrm{~m} / \mathrm{s}$.

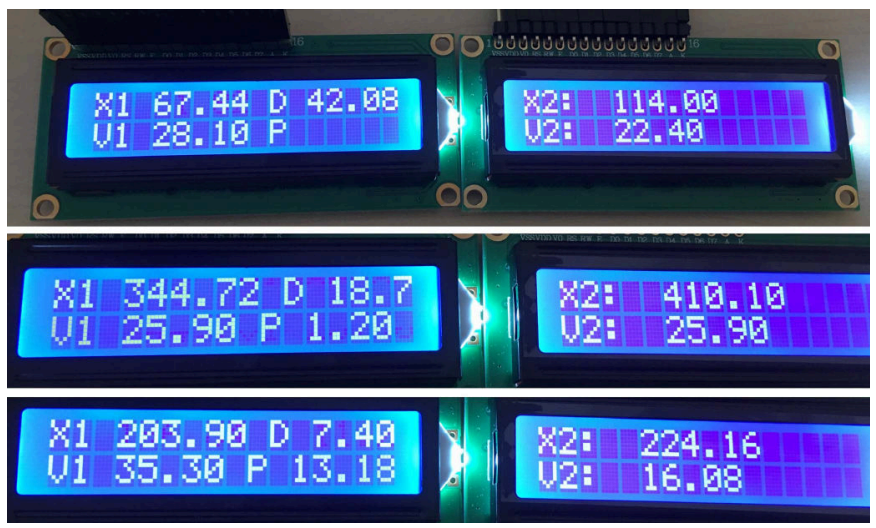

Fig. 11. Raspberry Pi displays, respectively, during approach, platooning and overtake. $\mathrm{X}$ is the position, $\mathrm{V}$ the velocity, $\mathrm{D}$ the distance between vehicles and $\mathrm{P}$ is the payment.

TABLE VIII

CALCUlation Times For Functions On RASPBERRy Pi

\begin{tabular}{lccc} 
Function & Average [s] & Std. Dev. & Coef. of Variation \\
\hline EvalPlat & $5.79 \cdot 10^{-4}$ & $2.2 \cdot 10^{-5}$ & $3.79 \cdot 10^{-2}$ \\
EvalOvt & 0.38 & $7.5 \cdot 10^{-3}$ & $1.9 \cdot 10^{-2}$
\end{tabular}

Raspberry Pi 3 Model B equipped with a 64bit ARM CortexA53 quad-core CPU with $1.2 \mathrm{GHz}$ frequency and $1 \mathrm{~GB}$ RAM. To demonstrate the negotiation, two boards have been used, connected by Ethernet and equipped with displays, as shown in Fig. 11.

The algorithm adheres to the activity diagram from Fig. 3, but is equipped with additional connectivity and synchronisation modules.

1) Computational Complexity: To assess the computational burden, the execution times of the algorithm were measured using the Raspberry Pi's clock_gettime() function. twenty observations were made for platooning and overtake scenarios. The time values obtained are shown in Tab. VIII. The platooning evaluation function takes less than $0.001 \mathrm{~s}$ to execute, while the overtake evaluation consistently less than $0.4 \mathrm{~s}$. Because the algorithm is to be executed once per manoeuvre, this example demonstrates its feasibility in real time application. 


\section{CONCLUSION}

This paper proposes the application of a game-theoretic cooperative-competitive approach to the interaction between CAVs, intending to improve road transport energy efficiency by introducing means to achieving payment-based self-enforced cooperation. The variation of agent parameters required to enable cooperation is derived from the proposed user-defined cost of time. User input may double as a means of matching vehicles driving style to user's preference. The algorithms to compute energy and time economy for cruising, platooning and overtaking have been provided, example calculations presented and a hardware-in-the-loop application demonstrated. Further work includes the implementation of an intersection model, a hardware-in-the-loop demonstrator on scaled, automated vehicles and development of the cost function to facilitate refined dynamics control, n-player game formulation.

\section{APPENDIX A POWERTRAIN MODEL}

To evaluate energy consumption and cost of the maneuvres, powertrains are modelled as a quasi-static backward facing model, following either the steady state velocity or the velocity profiles presented in Fig. 4 above.

The control variable is the acceleration $a_{n}$, being assumed constant, as dynamics are not a subject of this study. As the boundary velocities: $V_{n}^{*}$ and $V_{n}^{\text {ovt }}$, the time of the acceleration manoeuvre is found

$$
T_{a}=\frac{\left(V_{n}^{o v t}-V_{n}^{*}\right)}{a_{n}}
$$

and the time is discretised into $T=1,2, \ldots, i$ elements with a timestep $t_{\text {step }}$. The force required is found by relating to the balance of forces defined as

$$
F_{n e t, i, n}=F_{W, i, n}-F_{\text {roll }, i, n}-F_{\text {drag }, i, n}
$$

where the $F_{W, i, n}$ is the propulsive force, assumed to represent torque of the motor. The powertrain energy consumption is calculated as

$$
E_{i, n}=\frac{1}{\eta_{P}} F_{W, i, n} v_{i, n} t_{s t e p}
$$

where the powertrain efficiency $\eta_{P}$ is a product of all powertrain components $\eta_{i, M}, \eta_{i, P E}, \eta_{i, B}$, referring, respectively, to electric motor, power electronics and battery.

The force of rolling resistance is defined as

$$
F_{\text {roll }, i, n}=v_{i, n} \mu_{\text {roll }, i}
$$

and the aerodynamic drag force as

$$
F_{\text {drag }, i, n}=\frac{1}{2} A_{n} \rho_{a i r} C_{i, d} v_{i, n}^{2} .
$$

Vehicle's state is defined by its acceleration $a_{n}$, velocity $v_{i, n}$ and position $s_{i, n}$ as

$$
\begin{aligned}
a_{i, n} & =\frac{F_{n e t, i, n}}{m_{i}}, \\
v_{i+1, n} & =v_{i, n}+a_{i, n} t_{\text {step }}, \\
s_{i+1, n} & =s_{i, n}+v_{i, n} t_{\text {step }} .
\end{aligned}
$$

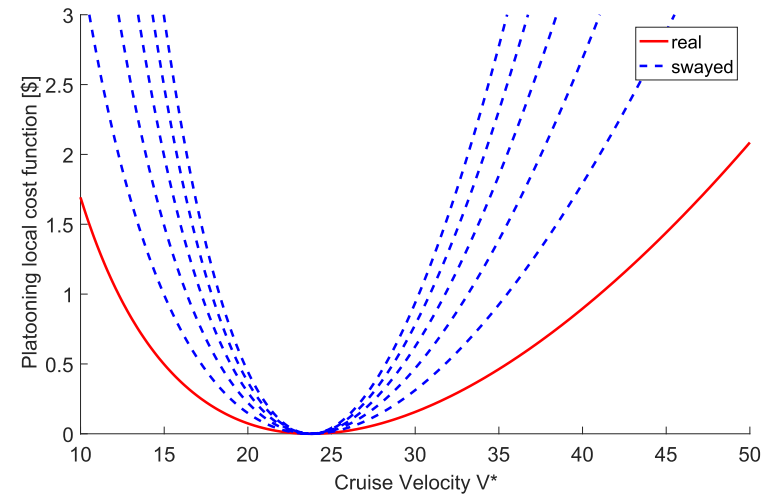

Fig. 12. Comparison of truthful and cheated cost function dynamics. $\mathfrak{X}=$ $\{2,3,4,5,6\}$.

Finally, the energy expense of a maneuver is found as

$$
E_{n}=\sum_{N} E_{i, n} .
$$

Friction breaking is neglected here, as any use of breaks is wasteful from the energy efficiency perspective, and hence, since the safety is neglected, optimal solutions would never use it. It may be introduced together with a battery state of charge and state of health models.

\section{APPENDIX B}

\section{Derivation of the DyNamic OVERTAKe Gap Length}

Equation (27) for the dynamic overtake gap length $S_{\max }$ has been derived from the real length of the gap $S_{g a p}$, velocity of the gap $V_{\text {gap }}$, assuming that the velocities of oncoming vehicles is constant. After factoring the $S_{\text {gap }}$ into the parentheses the equation (27) is

$$
S_{\text {max }}=S_{\text {gap }}-\frac{S_{\text {gap }}}{V_{E V}^{\text {ovt }}} V_{\text {gap }},
$$

where the fraction defines the overtake time

$$
T_{o v t}=\frac{S_{g a p}}{V_{E V}^{\text {ovt }}},
$$

which then allows to find the distance the oncoming traffic travels during the overtake

$$
S_{\text {oncoming }}=V_{\text {gap }} T_{\text {ovt }}
$$

which is substracted from the static gap, arriving back at (44).

\section{APPENDIX C PROBLEM OF HONESTY}

As the proposed framework features a payment system, agents may be incentivised to game the system to increase received payments. Thus to minimize legislative and regulatory effort to control the traffic, cheating possibilities are examined.

Scenarios' sensitivity to agent's dishonesty is considered.

1) Platooning: Given that agents request a payment for change in velocity, agents may increasing objective's slope, while maintaining the same minimizer. It is achievable if constant parameters of the cost function, as defined in (10b-d) are swayed by a constant cheating multiplier $\mathfrak{X}$. Problem's dynamics are presented in Fig. 12. This vulnerability can be mitigated, however, by demand to share the cruise cost $C_{C, n}$ as a control value. 
2) Overtake: An overtake is a dynamic event. The $C_{\text {ovt }}$ is sensitive to from vehicle mass (39), which cannot be inferred from observation. Therefore agents may be inclined to overvalue it as the payment is proportional to agent's mass

$$
p \propto C_{o v t}^{n} .
$$

However, since vehicle other parameters can be measured, a networked approach to cheating resilient system design could be considered in future.

\section{ACKNOWLEDGMENT}

The authors would like to thank the Engineering and Physical Sciences Research Council (EPSRC) and Arrival Ltd., for funding this work, as well as the anonymous reviewers, for insightful comments.

\section{REFERENCES}

[1] T. Litman, "Autonomous vehicle implementation predictions," Victoria Transp. Inst., Victoria, BC, Canada, Tech. Rep., 2017.

[2] S.-W. Kim et al., "Multivehicle cooperative driving using cooperative perception: Design and experimental validation," IEEE Trans. Intell. Transp. Syst., vol. 16, no. 2, pp. 663-680, Apr. 2015.

[3] L. Hobert, A. Festag, I. Llatser, L. Altomare, F. Visintainer, and A. Kovacs, "Enhancements of V2X communication in support of cooperative autonomous driving," IEEE Commun. Mag., vol. 53, no. 12, pp. 64-70, Dec. 2015.

[4] E.-K. Lee, M. Gerla, G. Pau, U. Lee, and J.-H. Lim, "Internet of Vehicles: From intelligent grid to autonomous cars and vehicular fogs," Int. J. Distrib. Sensor Netw., vol. 12, no. 9, pp. 1-14, 2016.

[5] R. Hult, M. Zanon, S. Gros, and P. Falcone, "Energy-optimal coordination of autonomous vehicles at intersections," in Proc. Eur. Control Conf. (ECC), Jun. 2018, pp. 602-607.

[6] B. Németh and P. Gáspár, "Design of vehicle cruise control using road inclinations," Int. J. Vehicle Auton. Syst., vol. 11, no. 4, p. 313, 2013

[7] J. Ploeg, B. T. M. Scheepers, E. van Nunen, N. van de Wouw, and H. Nijmeijer, "Design and experimental evaluation of cooperative adaptive cruise control," in Proc. 14th Int. IEEE Conf. Intell. Transp. Syst. (ITSC), Oct. 2011, pp. 260-265.

[8] A. Alam, B. Besselink, V. Turri, J. Martensson, and K. H. Johansson, "Heavy-duty vehicle platooning for sustainable freight transportation: A cooperative method to enhance safety and efficiency," IEEE Control Syst. Mag., vol. 35, no. 6, pp. 34-56, Dec. 2015.

[9] T. Shamir, "How should an autonomous vehicle overtake a slower moving vehicle: Design and analysis of an optimal trajectory," IEEE Trans. Autom. Control, vol. 49, no. 4, pp. 607-610, Apr. 2004.

[10] S. Tsugawa, S. Kato, T. Matsui, H. Naganawa, and H. Fujii, "An architecture for cooperative driving of automated vehicles," in Proc. IEEE Intell. Transp. Syst. (ITSC), Oct. 2000, pp. 422-427.

[11] G. Usman and F. Kunwar, "Autonomous vehicle overtaking-An online solution," in Proc. IEEE Int. Conf. Automat. Logistics, Aug. 2009, pp. 596-601.

[12] P. Petrov and F. Nashashibi, "Modeling and nonlinear adaptive control for autonomous vehicle overtaking," IEEE Trans. Intell. Transp. Syst., vol. 15, no. 4, pp. 1643-1656, Aug. 2014.

[13] N. Murgovski and J. Sjoberg, "Predictive cruise control with autonomous overtaking," in Proc. 54th IEEE Conf. Decis. Control (CDC), vol. 54, Dec. 2015 , pp. 644-649.

[14] M. Birdsall, "Google and ITE: The road ahead for self-driving cars," J. Inst. Transp. Eng., vol. 84, no. 5, pp. 36-39, 2014.

[15] M. Dikmen and C. M. Burns, "Autonomous driving in the real world: Experiences with tesla autopilot and summon," in Proc. 8th Int. Conf. Automot. User Interfaces Interact. Veh. Appl. (Automotive'UI), Oct. 2016, pp. 225-228.

[16] L. Makarem and D. Gillet, "Decentralized coordination of autonomous vehicles at intersections," IFAC Proc. Volumes, vol. 44, no. 1, pp. 13046-13051, 2011

[17] M. A. M. Zulkefli, J. Zheng, Z. Sun, and H. X. Liu, "Hybrid powertrain optimization with trajectory prediction based on inter-vehiclecommunication and vehicle-infrastructure-integration," Transp. Res. C, Emerg. Technol., vol. 45, pp. 41-63, Aug. 2014.

[18] Y. Jiang, M. Zanon, R. Hult, and B. Houska, "Distributed algorithm for optimal vehicle coordination at traffic intersections," IFACPapersOnLine, vol. 50, no. 1, pp. 11577-11582, Jul. 2017.
[19] R. Azimi, G. Bhatia, R. R. Rajkumar, and P. Mudalige, "STIP: Spatiotemporal intersection protocols for autonomous vehicles," in Proc. ACM/IEEE Int. Conf. Cyber-Phys. Syst. (ICCPS), Apr. 2014, pp. 1-12.

[20] I. H. Zohdy and H. A. Rakha, "Intersection management via vehicle connectivity: The intersection cooperative adaptive cruise control system concept," J. Intell. Transp. Syst., vol. 20, no. 1, pp. 17-32, Jan. 2016.

[21] D. Carlino, S. D. Boyles, and P. Stone, "Auction-based autonomous intersection management," in Proc. 16th Int. IEEE Conf. Intell. Transp. Syst. (ITSC), Oct. 2013, pp. 529-534.

[22] K. Kang and H. A. Rakha, "Game theoretical approach to model decision making for merging maneuvers at freeway on-ramps," Transp. Res. Rec., J. Transp. Res. Board, vol. 2623, no. 1, pp. 19-28, Jan. 2017.

[23] M. Wang, S. P. Hoogendoorn, W. Daamen, B. van Arem, and R. Happee, "Game theoretic approach for predictive lane-changing and car-following control," Transp. Res. C, Emerg. Technol., vol. 58, pp. 73-92, Sep. 2015.

[24] W. Yang, Z. Zhiyong, Y. Jianhua, and G. Lifen, "Static game approach for solving lane-merging conflict between autonomous vehicles," in Proc. IEEE Int. Conf. Intell. Transp. Eng. (ICITE), Aug. 2016, pp. 53-57.

[25] C. Dextreit and I. V. Kolmanovsky, "Game theory controller for hybrid electric vehicles," IEEE Trans. Control Syst. Technol., vol. 22, no. 2, pp. 652-663, Mar. 2014.

[26] T. Gu and J. M. Dolan, "On-road motion planning for autonomous vehicles," in Proc. Int. Conf. Intell. Robot. Appl. (ICIRA), Montreal, QC, Canada, no. 3, 2012, pp. 588-597.

[27] C. Cavoli, B. Phillips, T. Cohen, and P. Jones, "Social and behavioural questions associated with automated vehicles a literature review," Univ. College London Transp. Inst., London, U.K., Tech. Rep., Jan. 2017, pp. $1-124$.

[28] A. Waytz, J. Heafner, and N. Epley, "The mind in the machine: Anthropomorphism increases trust in an autonomous vehicle," J. Exp. Social Psychol., vol. 52, pp. 113-117, May 2014.

[29] S. O. Hansson, "Decision theory," Technology, vol. 19, no. 1, pp. 1-94, 2005.

[30] A. Diekmann, D. Helbing, and R. O. Murphy, "Conference program game theory and society," ETH Zürich, Comput. Social Sci., Cathedral, CA, USA, Tech. Rep., 2011.

[31] (2018). The Coin of Transportation, Blockchain-Based Transportation Protocol. [Online]. Available: https://dav.network/

[32] D. A. Hensher, "Measurement of the valuation of travel time savings," J. Transp. Econ. Policy, vol. 35, no. 1, pp. 71-98, 2001.

[33] B. Roberson and S. S. Pilot, "Fuel conservation strategies: Cost index explained," Boeing Aero Quart., vol. 2, pp. 26-28, Apr. 2007.

[34] A. Bressan, "Noncooperative differential games. A tutorial," Dept. Math., Penn State Univ., State College, PA, USA, Tech. Rep., 2010, pp. $1-80$.

[35] C. Daskalakis, P. W. Goldberg, and C. H. Papadimitriou, "The complexity of computing a Nash equilibrium," SIAM J. Comput., vol. 39, no. 1, pp. 195-259, Jan. 2009.

[36] A. Y. S. Lam, Y.-W. Leung, and X. Chu, "Autonomous-vehicle public transportation system: Scheduling and admission control," IEEE Trans. Intell. Transp. Syst., vol. 17, no. 5, pp. 1210-1226, May 2016.

[37] C. S. Carver and M. F. Scheier, Attention and Self-Regulation: A Controltheory Approach to Human Behavior. New York, NY, USA: SpringerVerlag, 2012.

[38] G. Mohan, F. Assadian, and S. Longo, "An optimization framework for comparative analysis of multiple vehicle powertrains," Energies, vol. 6, no. 10 , pp. $5507-5537,2013$

[39] A. Kalai and E. Kalai, "A cooperative value for Bayesian games," Center Math. Stud. Econ. Manage. Sci., Tech. Rep., 2010.

[40] UK Government. (2018). Energy Price Statistics. [Online]. Available: https://www.gov.uk/government/

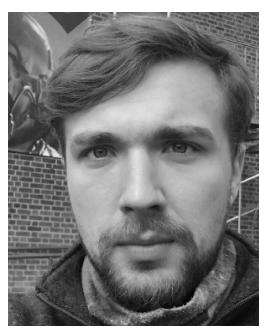

Marcin Stryszowski received the B.Eng. degree in power engineering from the Poznan University of Technology in 2012 and the double M.Sc. degrees in nuclear plant design from the Royal Institute of Technology (KTH) and ENSTA ParisTech in 2015. $\mathrm{He}$ is currently pursuing the Ph.D. degree in traffic energy optimization with Cranfield University. His M.Sc. thesis contributed to EDF Energy's nuclear safety research performing fluid dynamics of accident scenario. His research interests range from deterministic nuclear power safety to game-theoretic approach to conflict and cooperation modeling. 


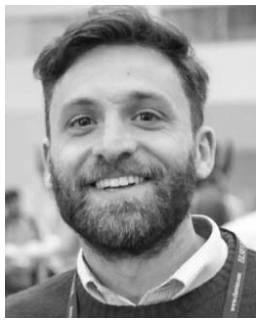

Stefano Longo (Senior Member, IEEE) received the M.Sc. degree in control systems from the University of Sheffield in 2007 and the Ph.D. degree in networked control systems from the University of Bristol in 2011. In November 2010, he was appointed to the position of Research Associate at Imperial College London. He is currently a Senior Lecturer (an Assistant Professor) in vehicle control with Cranfield University, and he has been there as an academic since 2012. He is the Course Director for the M.Sc. in automotive mechatronics and advanced motorsport mechatronics, a Chartered Engineer, an Associate Editor of Mechatronics Journal (Elsevier), an Elected Executive Member of IET Control and Automation Network and IET Automotive and Road Transport Systems Network, and a member of the IFAC Technical Committee on Mechatronic Systems and Automotive Control. In the last few years he has authored two books and over 70 research articles in peer-refereed journals and international conferences. His work and his research interests gravitate around the problem of implementing advanced control algorithms in hardware, where the controller design and the hardware implementation are not seen as two separate, decoupled problems, but as a whole. His Ph.D. thesis was awarded the Institution of Engineering and Technology (IET) Control and Automation Prize for significant achievements in the area of control engineering.

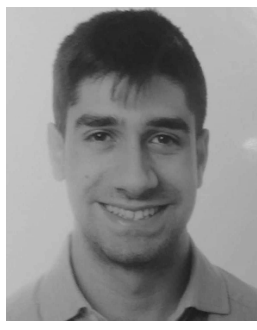

Dario D'Alessandro received the B.Sc and M.Sc degrees in computer science engineering from the Department of Electrical Engineering and Information Technology (DIETI), University of Naples Federico II (IT), in 2015 and 2018, respectively.

$\mathrm{He}$ has held the position of Visiting Research student in Transport Systems at the School of Aerospace, Transport and Manufacturing (SATM) at Cranfield University (UK) through the Erasmus+ in 2018, where he developed his M.Sc thesis project entitled "Hardware in the Loop validation of an optimal game theoretical approach for autonomous vehicles cooperation".

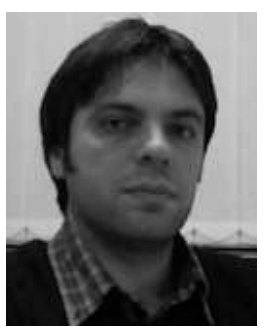

Efstathios Velenis (Senior Member, IEEE) received the M.Sc. and Ph.D. degrees from the School of Aerospace Engineering, Georgia Institute of Technology, GA, USA, in 2000 and 2006, respectively, and the Mechanical Engineering Diploma degree from the Mechanical Engineering Department, National Technical University of Athens, in 1999. In 2006, he was awarded the Luther Long Award for the Best Ph.D. Dissertation in engineering mechanics at the Georgia Institute of Technology. Following his Ph.D., he held a post-doctoral researcher position at the Georgia Institute of Technology and was a Visiting Researcher at Ford Motor Company, MI, USA. He is currently a Senior Lecturer with the Advanced Vehicle Engineering Centre, Cranfield University. His research focuses on control of vehicle dynamics, active chassis systems, and control of autonomous vehicles. He has coauthored more than 70 articles in peer-refereed journals and international conferences. He is an Editor of the IEEE TRANSACTIONS ON VEHICUlar TECHNOLOGY.

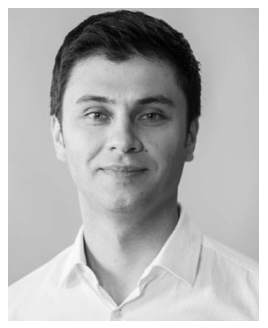

Gregory Forostovsky received the B.Eng. degree in automotive engineering with motorsport from the University of Hertfordshire in 2011 and the M.Sc. degree in power systems engineering from University College London (UCL) in 2016. He is currently working on a number of electric autonomous vehicle projects at Arrival Ltd., and has lead development of the autonomous Robocar and DevBot vehicles at Roborace Ltd.
Sabato Manfredi (Member, IEEE) has been an Assistant Professor of automatic control and an Adjunct Professor with the Department of Electrical Engineering and Information Technology, University of Naples Federico II, Italy, since 2005. He has been a Visiting Academic with the Control and Power Group, Electrical and Electronic Engineering Department, Imperial College, since 2012. His research interests are primarily in automatic control with a special emphasis on distributed optimization, embedded devices, sensor/drone networks, smart city, and Industry 4.0. He has published more than 80 scientific publications, including 18 single-author articles. He is the author of the book entitled Multilayer Control of Networked CyberPhysical Systems: Application to Monitoring, Autonomous and Robot Systems (Advances in Industrial Control Series, Springer 2017). He collaborates with many international universities and companies and holds European patent. $\mathrm{He}$ is a proponent member of an academic spin-off, and is involved in a range of academic, industrial, and consulting projects. 
2020-05-06

\section{A framework for self-enforced optimal interaction between connected vehicles}

Stryszowski, Marcin

IEEE

Stryszowski S, Longo S, D'Alessandro D, et al., (2020) A framework for self-enforced optimal interaction between connected vehicles. IEEE Transactions on Intelligent Transportation

Systems, Volume 22, Number 10, October 2021, pp. 6152-6161

https://doi.org/10.1109/TITS.2020.2988150

Downloaded from Cranfield Library Services E-Repository 